\title{
Erratum to: Limit computable integer parts
}

\author{
Paola D’Aquino · Julia Knight · Karen Lange
}

Published online: 28 February 2015

(C) Springer-Verlag Berlin Heidelberg 2015

\section{Erratum to: Arch. Math. Logic (2011) 50:681-695 DOI 10.1007/s00153-011-0241-z}

The authors would like to publish an erratum to address the error in Proposition 3.1 and its consequences. Proposition 3.1 in [1] was incorrect as stated. In particular, the purported implications $2 \Longrightarrow 1$ and $3 \Longrightarrow 1$ are false; there exists a real closed field $R$, a $Z$-ring $I \subset R$, and $a \in R-I$ such that $p(a)$ is infinite or in $\mathbb{Z}$ for all $p(x) \in I[x]$, but there is no Z-ring $J$ such that $I \subset J \subset R$ and $a \in J$. We thank Emil Jeřábek [2] for pointing out the error and providing the following counterexample.

\section{Counterexample to Proposition 3.1}

Let $R$ be the real closure of $\mathbb{Q}[x]$ where $x$ is an infinite element. Let $I \subset R$ be the subring $\left(x^{2}+1\right) \mathbb{Q}\left[x^{2}\right]+\mathbb{Z}$, and let $a=x$. The ring $I[x]$ is discrete: for an element $p(x)$ of $I[x]=I+x I$, either $p(x)$ is a non-constant polynomial from $\mathbb{Q}[x]$, in which case, it is infinite, or $p(x)$ is an integer. Hence, conditions 2 and 3 of Proposition 3.1 are satisfied. It is also easy to see that $I$ is a $Z$-ring. On the other hand, $I[x]$ contains the ring $\mathbb{Z}\left[x,\left(x^{2}+1\right) / 4\right]$ from Example 2, which does not extend to any $Z$-ring. Hence $I$ cannot be extended to any Z-ring containing $a=x$.

The online version of the original article can be found under doi:10.1007/s00153-011-0241-z.

\section{J. Knight · K. Lange ( $\varangle)$}

Department of Mathematics, University of Notre Dame, 255 Hurley, Notre Dame, IN 46556, USA e-mail: klange1@nd.edu

P. D’Aquino

Dipartimento di Matematica, Seconda Universita' di Napoli, via Vivaldi, 43, Caserta 81100, Italy e-mail: Paola.daquino@unina2.it 
By adding an additional hypothesis to Proposition 3.1, we can salvage a version of the result (which appears in [3]). This revised version of Proposition 3.1 suffices to prove the main result of the paper Theorem 3.5 (as discussed below).

Proposition 3.1' (Revised Proposition 3.1) Suppose $R$ is a real closed field, $I$ is a $Z$-ring such that $I \subseteq R$, and $a \in R-I$.

(1) Suppose there is a Z-ring $J$ such that $I \subseteq J \subseteq R$ and $a \in J$. Then,

(a) for all $p(x) \in I[x], p(a)$ is either infinite or in $\mathbb{Z}$, so

(b) for all $p(x) \in I[x], p(a) \notin(0,1)$.

(2) If $p(a)$ is infinite for all nonconstant $p(x) \in I[x]$, then there is a Z-ring $J$ such that $I \subseteq J \subseteq R$ and $a \in J$.

Proof The proof of the first statement is given in the original paper. We must prove the second statement. We let $J \subset R$ consist of the elements of the form $\frac{p(a)}{n}+z$, where $p(x) \in I[x]$ has constant term $0, n \in \mathbb{N}$, and $z \in I$. We first show that $J$ is discrete. Let $\frac{p(a)}{n}+z \in J$. If $p(x)$ is the zero polynomial, then $\frac{p(a)}{n}+z=z$. Since $I$ is discrete, $z \notin(0,1)$. Otherwise, since $p(x)+n z \in I[x]$ is a nonconstant polynomial, $p(a)+n z$ is infinite by assumption. Then, $\frac{p(a)+n z}{n}=\frac{p(a)}{n}+z$ is infinite as well, so $J$ is discrete. Next, we show that $J$ is a $Z$-ring. Given an element $\frac{p(a)}{n}+z$ and $m \in \mathbb{N}$, we have $\frac{p(a)}{n}+z=\frac{p(a)}{n m} \cdot m+z^{\prime} m+k$, where $z=z^{\prime} m+k$ with $z^{\prime} \in I$ and $0 \leq k<m$. We have such a $z^{\prime} \in I$ and $k \in \mathbb{N}$ because $I$ is a $Z$-ring. Then, $\frac{p(a)}{n}+z=\left(\frac{p(a)}{n m}+z^{\prime}\right) \cdot m+k$ with $0 \leq k<m$, so $J$ is a $Z$-ring.

The incorrect portion of Proposition 3.1 is used in Theorem 3.2, Lemma 3.4, and Theorem 3.5. In Lemma 3.4 and Theorem 3.5, Statement (2) of Proposition 3.1' above suffices for the original proofs, as in each application we have that $p(a)$ is infinite for all nonconstant $p(x) \in I[x]$ for the appropriate element $a$ and $Z$-ring $I$.

The proof of Theorem 3.2 in the original paper, that every countable real closed field $R$ has a maximal $Z$-ring that is $\Delta_{2}^{0}(R)$ also relied on the incorrect version of Proposition 3.1. It is now unclear whether Theorem 3.2 is true, as Proposition 3.1' does not appear to suffice in this case. We are left with the following question.

Question 1 If $R$ is a countable real closed field, is there a maximal Z-ring $I \subseteq R$ such that I is $\Delta_{2}^{0}(R)$ ?

This question is interesting in its own right. However, by Theorem 3.5, a maximal $Z$ ring for $R$ may not be an integer part for $R$. Thus, finding an affirmative answer to Question 1 would not directly provide a $\Delta_{2}^{0}(R)$-integer part for $R$, the original motivating problem for this work.

Acknowledgments The authors would like to thank Salma Kuhlmann, David Marker, and Mojtaba Moniri for useful discussions and pointing out Example 2. The authors again thank Emil Jeřábek for pointing out the error. The second author received support from Seconda Universita' di Napoli and Istituto Nazionale di Alta Matematica. The third author was partially supported by National Science Foundation Grants DMS0802961 and DMS-1100604. 


\section{References}

1. D’Aquino, P., Knight, J., Lange, K.: Limit computable integer parts. Arch. Math. Logic 50(7-8), 681-695 (2011)

2. Jeřábek, E.: Personal correspondence

3. Wilkie, A.: Some results and problems on weak systems of arithmetic. In: Logic Colloquium '77. North Holland 